\title{
ON THE NON-MINIMAL MARTIN BOUNDARY POINTS
}

\author{
TERUO IKEGAMI
}

Dedicated to Professor Kiyoshi Noshiro on his 60th birthday

1. In a Green space ${ }^{11} \Omega$ we can introduce Martin's topology and make it the Martin space $^{2)} \hat{\Omega} . \quad \Omega$ is a dense open subset of $\hat{\Omega}$ and the kernel

$$
K(p, x)=\left\{\begin{array}{cl}
\frac{G(p, x)}{G\left(p, y_{0}\right)} & p \neq y_{0} \\
0 & p=y_{0} \neq x \\
1 & p=y_{0}=x
\end{array}\right.
$$

can be extended continuously to $(p, x) \in \hat{\Omega} \times \Omega$, where $G(p, x)$ is a Green function in $\Omega$ and $y_{0}$ the fixed point of $\Omega . \quad \hat{\Omega}$ is a metric space. $\Delta=\hat{\Omega}-\Omega$ is divided into two disjoint subsets $\Delta_{0}, \Delta_{1}$ and $s \in \Delta_{1}$ is characterized by the fact that $K(s, x)$ is a minimal positive harmonic function ${ }^{3)}$ in $x \in \Omega$.

2. We shall show the following theorem:

THEOREM. No point of $\Delta_{0}$ is an isolated point.

Proof. Let $\omega$ be an open subset of $\Omega,\left\{x_{n}\right\}(n=1,2, \ldots)$ be a sequence of points in $\omega$ such that $x_{n} \rightarrow x_{0} \in \Delta$. If we denote by $\mathscr{H}$ the family of positive superharmonic functions in $\Omega$, each of which dominates $K\left(x_{n}, y\right)$ on $\Omega-\omega$, then $\inf v(y)$ is equal to the positive superharmonic function except a polar set. We shall write this superharmonic function $\mathscr{C}_{K_{n}}^{\prime \prime \prime}(y)$.

In this case

Received June 17, 1966.

1) M. Brelot, G. Choquet, Espaces et lignes de Green. Annales Inst. Fourier 3 (1951), pp. 199-263.

2) M. Brelot, Le problème de Dirichlet. Axiomatique et frontière de Martin. Journal de Math. 35 (1956), pp. 297-335 (pp. 329-330). Cf. also R. S. Martin, Minimal positive harmonic functions, Trans. Amer. Math. Soc., 49 (1941), pp. 137-172. M. Parreau, Sur les moyennes des fonctions harmoniques et analytiques et la classification des surfaces de Riemann, Annales Inst. Fourier 3 (1952), pp. 103-197. L. Naïm, Sur le rôle de la frontière de R. S. Martin dans la théorie du potentiel, Annales Inst. Fourier 7 (1957), pp. 183-281.

3) R. S. Martin, loc. cit., p. 137. 


$$
\mathscr{C}_{K_{n}}^{w}(y)=\int K(x, y) d \mu_{n}(x)
$$

where $\mu_{n}$ is a positive mass-distribution on $\omega \cap \Omega$ and the total mass of $\mu_{n}$ does not exceed 1, $\omega$ being the boundary of $\omega$ in $\hat{\Omega}$. By the theorem of choice, we can extract from $\left\{\mu_{n}\right\}$ the subsequence $\left\{\mu_{n}^{\prime}\right\}$ such that $\mu_{n}^{\prime}$ converges vaguely to $\mu$ and the carrier of $\mu$ is contained in $\bar{\omega} \cap \Omega$.

$$
v(y)=\int K(x, y) d \mu(x)
$$

is a positive superharmonic function in $\Omega$, and we have

$$
\mathscr{C}_{K_{x_{0}}}^{w}(y) \leq v(y) \text {. }
$$

In fact, for fixed $y \in \Omega$ and $r>0$ we shall denote by $\varepsilon_{y}^{\prime r}$ the mass-distribution which can be obtained after sweeping out the unit mass on $y$ to the exterior of the sphere (circle) of radius $r$ and with center $y$. Then

$$
U^{r}(x)=\int K(x, z) d \varepsilon_{y}^{\prime r}(z)
$$

is bounded and continuous on $\hat{\Omega}$. Therefore

$$
\lim _{n \rightarrow \infty} \int U^{r}(x) d \mu_{n}^{\prime}(x)=\int U^{r}(x) d \mu(x) .
$$

By reciprocal law

$$
\lim _{n \rightarrow \infty} \int \mathscr{E}_{K_{x n^{\prime}}}^{\omega}(z) d \varepsilon_{y}^{\prime r}(z)=\int v(z) d \varepsilon_{y}^{\prime \prime}(z)
$$

and by Fatou's lemma

$$
\int \mathscr{C}_{K_{x_{0}}}^{\omega}(z) d \varepsilon_{y}^{\prime r}(z) \leq \lim _{n \rightarrow \infty} \int \mathscr{C}_{K_{x_{n^{\prime}}}}^{\omega}(z) d \varepsilon_{y}^{\prime r}(z)=\int v(z) d \varepsilon_{y}^{\prime r}(z) .
$$

By making $r \rightarrow 0$ we can get for each $y \in \Omega$

$$
\mathscr{C}_{K_{x_{0}}}^{w}(y) \leq v(y) .
$$

If $\mu_{1}$, denotes the restriction of $\mu$ to $\Delta$ and $\mu_{2}$ the restriction of $\mu$ to $\Omega$, then

$$
\begin{aligned}
v(y) & =\int K(x, y) d \mu_{1}(x)+\int K(x, y) d \mu_{2}(x) \\
& =u(y)+w(y)
\end{aligned}
$$

where $u$ is harmonic and $w$ is a potential, and this is just the Riesz decomposi- 
tion.

From now on let $x_{0}$ be a point of $\Delta_{0}$ and $x_{0}$ be isolated. Let

$$
K\left(x_{0}, y\right)=\int_{\Delta_{1}} K(x, y) d \nu(x)
$$

be the canonical representation " of $K\left(x_{0}, y\right)$. Then we can find a neighbourhood $\hat{\delta}$ of $x_{0}$ such that

$$
\overline{\hat{\delta}} \cap \Delta_{0}=\left\{x_{0}\right\}
$$

and

$$
\nu\left(\Delta_{1}-\overline{\hat{\delta}}\right)>0
$$

If we set $\omega=\hat{\delta} \cap \Omega$, then

$$
\begin{aligned}
\mathscr{E}_{K_{x_{0}}}^{\omega}(y) & =\int_{\Delta_{1}} \mathscr{E}_{K_{x}}^{\omega}(y) d \nu(x) \\
& =\int_{\Delta_{1}-\hat{\delta}}-\mathscr{C}_{K_{x}}^{\omega}(y) d \nu(x)+\int_{\hat{\hat{\delta}} \cap \Delta_{1}} \mathscr{E}_{K_{x}}^{\omega}(y) d \nu(x)
\end{aligned}
$$

The first term of the last side is harmonic, because $\omega$ is thin at each point of $\Delta_{1}-\bar{\delta}^{5 !}$ and therefore we can get $\mathscr{C}_{K_{x}}^{(1)}(y) \equiv K(x, y)$.

We note that $\mu_{1}$ is the restriction of the mass-distribution $\mu$ to $(\overline{\hat{\delta} \cap \Omega}) \cap \Delta$, which is contained in $\bar{\delta} \cap \Delta$ and does not contain the point $x_{0}$. By (1) we can get $\mu_{1}\left(\Delta_{0}\right)=0$, that is, $\mu_{1}$ is the canonical mass-distribution of $u$, and by (2)

$$
u_{1}(y)=\int_{\Delta_{1}-\overline{\hat{\delta}}} K(x, y) d \nu(x)>0
$$

Since

$$
\begin{aligned}
v(y) & =u(y)+w(y) \\
& \geq \mathscr{C}_{K_{x_{0}}}^{\omega}(y) \geq \int_{\Delta_{1}-\hat{\delta}}-\mathscr{C}_{K_{x}}^{\omega}(y) d \nu(x)=\int_{\Delta_{1}-\overline{\hat{\delta}}} K(x, y) d \nu(x)=u_{1}(y)
\end{aligned}
$$

and $u$ is the greatest harmonic minorant of $v$, we have

$$
u \geq u_{1},
$$

but the canonical mass-distribution of $u$ has the carrier in $\Delta_{1} \cap \overline{\hat{\delta}}$, whereas the canonical mass-distribution of $u_{1}$ has positive mass only in $\Delta_{1}-\overline{\hat{\delta}}$. As $u_{1}>0$

4) R. S. Martin, loc. cit., p. 157.

5) L. Naïm, loc. cit., p. 203 (théorème 3) and p. 205 (théorème 5). 
the canonical mass-distribution of $u$ has positive mass in $\Delta_{1}-\overline{\hat{\delta}}$; this is a contradiction.

Q.E D.

Corollary. If $\Delta_{0} \neq \phi$ then $\Delta_{0}$ contains at least countable points.

Remark. In the above consideration we rely upon the following argument: we have always $u \geq u_{1}$, and, if $u_{1}>0$, then $\mu_{1}$ is not the canonical mass-distribution of $u$.

Mr. K. Matsumoto has kindly pointed out the following result:

Let $x_{0}$ be a point of $\Delta_{0}$ and $\nu$ be the canonical mass-distribution of $K_{x_{0}}(y)$, then the common part of the carrier of $\nu$ with $\Delta_{1}$ is contained in $\bar{\Delta}_{0}$.

The proof follows from the preceding remark; let $E$ be the carrier of $\nu$. If $E \cap \Delta_{1} \nsubseteq \bar{\Delta}_{0}$, then there exist a point $z_{0}$ and a set $A$ satisfying the following conditions :

1) $A$ is an open neighbourhood of $z_{0}$ in $\Delta$,

2) $\nu(A)>0$,

3) $A \subset \Delta_{1}$.

We can construct an open set (in $\hat{\Omega}$ ) $G: G \cap \Delta=A$. In this case, there exist two positive numbers $0<\rho_{1}<\rho$ such that:

i) dist $\left(z_{0}, x_{0}\right)>\rho$,

ii) $\overline{U_{\rho}\left(z_{0}\right)} \subset G^{6}$,

iii) $\nu\left(U_{p_{1}}\left(z_{0}\right) \cap \Delta\right)>0$.

If we set $\omega=\Omega-\overline{U_{p}\left(z_{0}\right)}$, under the same notations as in the proof of the above theorem, we see from i), $x_{0} \in \bar{\omega}$ and, as $\overline{(\omega \cap \Omega)} \cap \Delta \subset \overline{U_{\rho}\left(z_{0}\right)} \cap \Delta \subset G \cap \Delta=A \subset \Delta_{1}$, $\mu_{1}$ is canonical and from $\Delta-\bar{\omega} \supset U_{p_{1}}\left(z_{0}\right) \cap \Delta, u_{1}>0$, this is a contradiction.

\section{Osaka City University}

6) We denote $U_{\rho}\left(z_{0}\right)=\left\{x \in \hat{\Omega} ;\right.$ dist $\left.\left(x, z_{0}\right)<\rho\right\}$, where the metric dist $\left(x, z_{0}\right)$ is the Martin's metric. 